\title{
Prior myocarditis and ventricular arrhythmias: The importance of scar pattern (e)
}

\author{
Michela Casella, MD, PhD, ${ }^{* \dagger}$ Marco Bergonti, MD, ${ }^{\star \S}$ Maria Lucia Narducci, MD, \\ Simone Persampieri, MD, ${ }^{*}$ Alessio Gasperetti, MD, ${ }^{*} \|$ Edoardo Conte, MD, * \\ Valentina Catto, PhD, ${ }^{*}$ Corrado Carbucicchio, MD, * Federico Guerra, MD, ${ }^{\dagger * *}$ \\ Gianluca Pontone, MD, ${ }^{*}$ Daniele Andreini, MD, PhD, ${ }^{\star \S}$ Cristina Basso, MD, PhD, ${ }^{\dagger \dagger}$

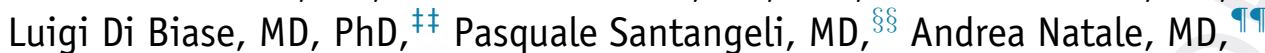 \\ Gemma Pelargonio, MD, Antonio Dello Russo, MD, PhD, ${ }^{\dagger * * 1}$ \\ Claudio Tondo, MD, PhD*\$1
}

From the *Centro Cardiologico Monzino, IRCCS, Milano, Italy, ${ }^{\dagger}$ Cardiology and Arrhythmology Clinic, University Hospital "Ospedali Riuniti Umberto I-Lancisi-Salesi”, Ancona, Italy, "Department of Clinical, Special and Dental Sciences, Marche Polytechnic University, Ancona, Italy, ${ }^{\S}$ Department of Biomedical, Surgical and Dental Sciences, University of Milan, Milano, Italy, ${ }^{\mathfrak{T}}$ Fondazione Policlinico Universitario A Gemelli, IRCCS, Rome, Italy, "University Heart Center, University Hospital Zurich, Zürich, Switzerland, **Department of Biomedical Science and Public Health, Marche Polytechnic University, Ancona, Italy, ${ }^{\dagger}$ Cardiovascular Pathology Unit, Department of Cardiac, Thoracic, Vascular Sciences and Public Health, Azienda Ospedaliera-University of Padua, Padova, Italy, ${ }^{*}$ Albert Einstein College of Medicine, Montefiore Hospital, Bronx, New York, ${ }^{\S \S}$ Division of Cardiology, Hospital of the University of Pennsylvania, Philadelphia, Pennsylvania, and ${ }^{\boldsymbol{\top} \Phi}$ Texas Cardiac Arrhythmia Institute, St. David's Hospital, Austin, Texas.

BACKGROUND Multiple studies have addressed the importance of anteroseptal scar in patients with nonischemic cardiomyopathy. However, this pattern has never been fully evaluated in patients with prior myocarditis.

OBJECTIVE The purpose of this study was to evaluate whether anteroseptal scar is associated with worse outcome in patients with prior myocarditis and how it affects the efficacy of catheter ablation (CA).

METHODS This was a retrospective study of consecutive patients with prior myocarditis and arrhythmic presentation. Cardiac magnetic resonance and electroanatomic voltage mapping were used to identify the scar pattern. Patients were referred for either CA or escalated antiarrhythmic drug (AAD) therapy. The main outcome was ventricular arrhythmia (VA)-free survival according to the presence of anteroseptal scar.

RESULTS A total of 144 consecutive patients with prior myocarditis were included. Mean age was $42.1 \pm 14.9$ years, and $58 \%$ were men.
Ejection fraction was normal in $73 \%$ of patients. Anteroseptal scar was present in $44 \%$ of cases. Sixty-one patients (42\%) underwent CA. Overall, at 2-year follow-up, VA-free survival was $77 \%$ in the CA group. After $C A$, the mean number of AADs taken by each patient decreased from 1.8 to $0.9 /$ die $(p<0.001)$. The presence of anteroseptal scar was found to be an independent predictor of VA relapse both in patients treated with CA (hazard ratio [HR] 3.6; 95\% confidence interval $[\mathrm{CI}] 1.1-11.4 ; P=.03)$ and in the overall population (HR 2.0; 95\% CI 1.2-3.5; $P=.02$ ) .

CONCLUSION In patients with prior myocarditis and VA, the presence of anteroseptal scar negatively predicts outcomes irrespective of treatment strategy.

KEYWORDS Anteroseptal scar; Cardiac magnetic resonance; Catheter ablation; Electroanatomic voltage mapping; Prior myocarditis; Ventricular arrhythmia

(Heart Rhythm 2021; $\mathbf{\square}: 1-8)$ (c) 2020 Heart Rhythm Society. All rights reserved.

Funding sources: This research did not receive any specific grant from funding agencies in the public, commercial, or not-for-profit sectors. Disclosures: Dr Di Biase is a consultant for Biosense Webster, Boston Scientific, Stereotaxis, Rhythm Management, and Abbott; and has received speaking honoraria from Medtronic, Pfizer, Bristol Myers Squibb, and Biotronik. Dr Natale is a consultant for Boston Scientific, Biosense Webster, St. Jude Medical, Biotronik, and Medtronic. Dr Tondo has received modest honoraria from St. Jude Medical and Abbott; and serves as member of the advisory board for Medtronic, Inc., and Boston Scientific Corp. All other authors have reported that they have no relationships relevant to the contents of this paper to disclose. ${ }^{1} \mathrm{Dr}$ Claudio Tondo and Dr Antonio Dello Q2 Russo share senior authorship. Address reprint requests and correspondence: Dr Marco Bergonti, Centro Cardiologico Monzino, IRCCS, Via C. Parea, 4, 20138 Milano, Italy. E-mail address: bergman21@gmail.com. 


\section{Introduction}

Patients with prior myocarditis often are young and have preserved left ventricular (LV) ejection fraction (EF). ${ }^{1}$ Nonetheless, they experience a high rate of malignant ventricular arrhythmias (VAs) (10\% per year), and the proportion of sudden cardiac deaths attributed to myocarditis at autopsy varies between $<10 \%$ and $20 \% .^{1,2}$ Importantly, apart from ventricular tachycardia (VT) and ventricular fibrillation, nonsustained ventricular tachycardia (NSVT) and frequent premature ventricular complexes (PVCs) are also associated with increased cardiovascular mortality in this setting.

Based on current guidelines, medical therapy is the standard of care for treating arrhythmic complications of myocarditis. ${ }^{4,5}$ However, it is not rare that medical therapy fails to control the arrhythmic burden in these patients. Previous studies demonstrated that radiofrequency catheter ablation (CA) can be effective in controlling drug-refractory VT. ${ }^{6-8}$ Nevertheless, evidence supporting the benefit of CA mainly stems from small retrospective studies. In the absence of large comparative studies, limited data support the safety and long-term efficacy of CA in this setting.

Multiple studies have addressed the importance of anteroseptal scar in patients with nonischemic cardiomyopathy (NICM) and acute myocarditis. ${ }^{1,9-12}$ However, this pattern has never been specifically evaluated in patients with prior myocarditis, and its impact on CA outcomes is unknown. $^{13-15}$

This study aimed to (1) determine the predictors of VA recurrence with special focus on the underlying scar substrate; and (2) evaluate the efficacy of CA in a large cohort of consecutive patients affected by prior myocarditis with arrhythmic presentation.

\section{Materials and methods Study design}

This study was a retrospective analysis of prospectively collected clinical data on all consecutive patients with prior myocarditis and VA referred to the electrophysiology departments of 2 tertiary referral centers for VA in Italy-Centro Cardiologico Monzino (Milan) and Policlinico Agostino Gemelli (Rome)—between 2010 and 2019.

To establish a diagnosis of prior myocarditis, all the following criteria had to be fulfilled: (1) history of endomyocardial biopsy (EMB)-proved (Dallas criteria) or cardiac magnetic resonance (CMR)-proved (Lake Louise criteria) acute myocarditis more than 12 months before the index evaluation $^{5,14}$; (2) replacement fibrosis at EMB (when available) or presence of nonischemic late gadolinium enhancement (LGE) consistent with prior myocarditis at CMR; (3) no electrocardiographic (ECG), echocardiographic, or CMR criteria suggesting other cardiomyopathies; and (4) absence of significant coronary artery stenosis at coronary angiogram or computed tomography scan. ${ }^{16-20}$ The presence of active myocarditis, demonstrated by EMB (leukocyte infiltration), CMR (edema), or laboratory examination (high serum troponin level) constituted an exclusion criteria.
CMR protocols for image acquisition as well as EMB protocols for histologic processing are given in Supplemental Appendixes 1 and 2.

VA was defined as the presence of ventricular fibrillation, sustained VT, symptomatic NSVT/frequent PVCs $(>10,000 /$ $10 \% /$ die), or asymptomatic NSVT/PVCs presumed to cause ${ }_{\mathrm{Q} 6}$ ventricular dysfunction. Antiarrhythmic medications were defined in accordance with the Vaughan Williams classification. $^{21}$

All patients who satisfied the diagnostic criteria for prior myocarditis and had VA were included in the analysis. Upon clinical indication and based on current guideline recommendations, patients were referred for either CA (CA group) or escalated antiarrhythmic drug therapy (AAD group). ${ }^{3-5}$ Specifically, patients referred for CA were those with monomorphic VA and those in whom AAD was not tolerated, contraindicated, or already at its maximal dosage. The CA protocol is given in Supplemental Appendix 3. All EMB procedures were guided by endocavitary electroanatomic voltage mapping (EVM) acquired with the CARTO (Biosense Webster) or NavX (Abbott) system. EVM allows Q7 for 3-dimensional cardiac chamber reconstruction and recording of intracardiac ECGs. These combined data are represented on an EVM on which it is possible to localize areas of low voltage representing diseased tissue. The EMB protocol is extensively discussed in Supplemental Appendix $4 .^{22}$

The study protocol conforms to the ethical guidelines of the Declaration of Helsinki. All patients provided informed consent for all tests and procedures performed during hospitalization. The study protocol was approved by the Centro Cardiologico Monzino and Policlinico Agostino Gemelli Ethics Committee.

\section{Scar characterization}

Scar pattern was classified according to CMR evaluation with LGE when available and unipolar and bipolar EVM in the remaining cases. Two distinct scar patterns were identified: those with anteroseptal involvement $(\mathrm{S}+$ ) (at least $>30 \%$ of anteroseptal segments involved) and those without anteroseptal involvement (S-). Examples of anteroseptal scar and inferolateral scar are shown in Figures 1 and 2. The 2 groups ( $\mathrm{S}+$ and $\mathrm{S}-$ ) were compared for baseline characteristics, procedural data, and primary and secondary endpoints at longterm follow-up.

\section{Follow-up and outcomes}

After hospital discharge, follow-up was performed, consisting of outpatient visits every 6 months and 24-hour Holter ECG monitoring scheduled at 3, 6, and 12 months, and every 6 months thereafter. Patients with an implantable cardioverter-defibrillator (ICD) were reviewed every 6 months to retrieve stored ICD electrogram information. When longitudinal office follow-up visits were not available, telephone interviews were performed. Long-term outcomes included (1) survival free from any VA; and (2) mortality. 
VA recurrence was defined as the recurrence of the index VA or of any sustained VA. Relationships between the treatment strategy and outcomes were analyzed. Additionally, predictors of VA recurrence (with special attention to scar pattern) were investigated.

\section{Statistical analysis}

Continuous variables are given as mean $\pm \mathrm{SD}$ when normally distributed or as median (interquartile range) when nonnormally distributed. Accordingly, comparison between groups was made using the parametric (Student $t$ test) or nonparametric (Mann-Whitney $U$ test) test, respectively. Categorical variables are given as absolute value and percentage. Comparison between categorical variables was performed using the $\chi^{2}$ test and the Fisher exact test, as indicated. Eventfree survival was estimated by the Kaplan-Meier method using the log-rank test. Univariate Cox proportional hazard analysis assessed the relationship between baseline characteristics and procedural data with respect to arrhythmia recurrence. SPSS 23.0 (IBM Corp., Armonk, NY) was used for all statistical analyses. $P<.05$ for a 2 -tailed test was considered significant. Confidence intervals (CIs) were set at $95 \%$.

\section{Results}

\section{Baseline characteristics}

The final study population consisted of 144 patients (71 from Policlinico Agostino Gemelli and 73 from Centro Cardiologico Monzino). Baseline patient characteristics are summarized in Table 1. Mean patient age was $42.1 \pm 14.9$ years, and $83(57.6 \%)$ were male. The diagnosis of prior myocarditis was confirmed by EMB in 112 patients (77.8\%). Forty-eight patients $(35.8 \%)$ already had an ICD. Before ablation, antiarrhythmic therapy consisted of a mean of $1.63 \pm 0.56$ AADs; 46 (42.2\%) were taking amiodarone and $85(78.0 \%)$ were taking beta-blockers (Supplemental Figure S1 and Supplemental Table S1).

Seventy-three percent of patients had normal LV EF, and only $11(7.6 \%)$ had $\mathrm{EF}<35 \%$. According to pooled CMR and EVM evaluation, a pathologic substrate was identified in the anteroseptal region in 64 patients (44.4\%). Among these 64 patients, the diagnosis of prior myocarditis was confirmed by EMB in 55 (85.9\%). EVM- and CMRspecific results are given in Supplemental Figure S2.

The VAs for which patients were referred are listed in Table 1. The cumulative arrhythmic burden of the patients is given in Supplemental Table S2. Patients referred for CA were more often affected by VT, whereas patients referred because of resuscitated sudden cardiac death or ICD shock were more frequently managed with medical therapy (Supplemental Figure S3D). One-third of the patients affected by PVCs who were included in the study were referred because of suspicion of PVC-induced cardiomyopathy (low EF) superimposed on prior myocarditis. The remaining patients were referred because of symptoms. Additionally, patients in the CA group took more AADs at baseline (1.76 vs $1.45 ; P=.008)$, especially amiodarone
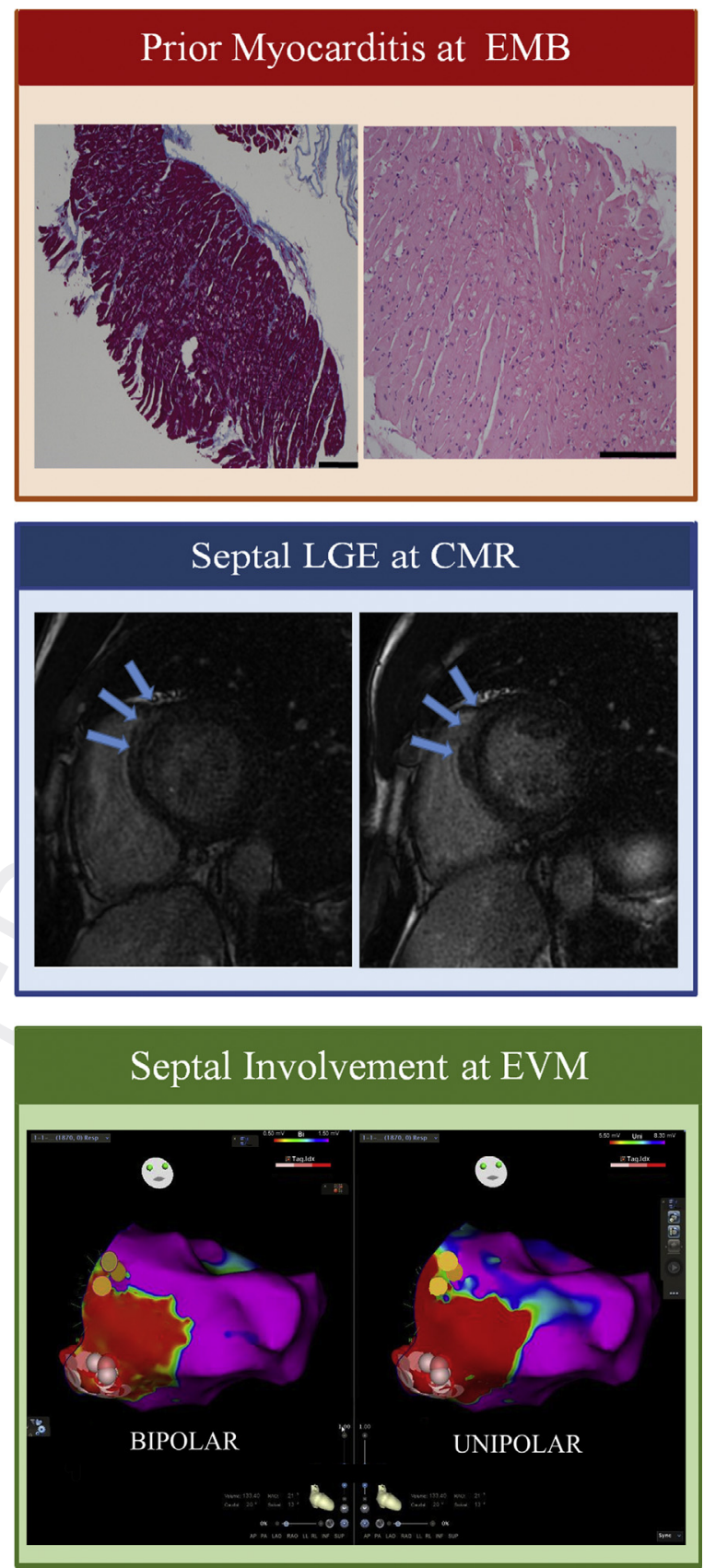

Figure 1 Anteroseptal scar. Top: Endomyocardial biopsy (EMB). Samples obtained from septum. Left: Masson trichrome staining shows spots of substitutive fibrosis and areas of damaged, possibly necrotic, cardiomyocytes (light red). Right: Hematoxylin-eosin staining shows cardiomyocytes of variable dimensions (between 15 and $21 \mu \mathrm{m}$ ), occasionally presenting perinuclear aloes, and cytoplasmic vacuolization. Middle: Cardiac magnetic Q8 resonance (CMR). Short-axis view of left ventricle shows septal hyperintensity signal on late gadolinium enhancement (LGE) images (arrows). Bottom: Endocardial electroanatomic voltage mapping (EVM). Data from a patient with anteroseptal scar showing (from left to right) endocardial bipolar and unipolar 3-dimensional EVM. Yellow dots represent the His and conduction system. White to red dots represent ablation sites.

(58.2\% vs $25.9 \%, P=.001)$ and Class IC AADs $(26.8 \%$ vs $6.6 \%, P=.005$ ) (Supplemental Table S1). Conversely, the 2 groups were homogeneous with regard to clinical characteristics, imaging data, and EVM analysis (Table 1). 

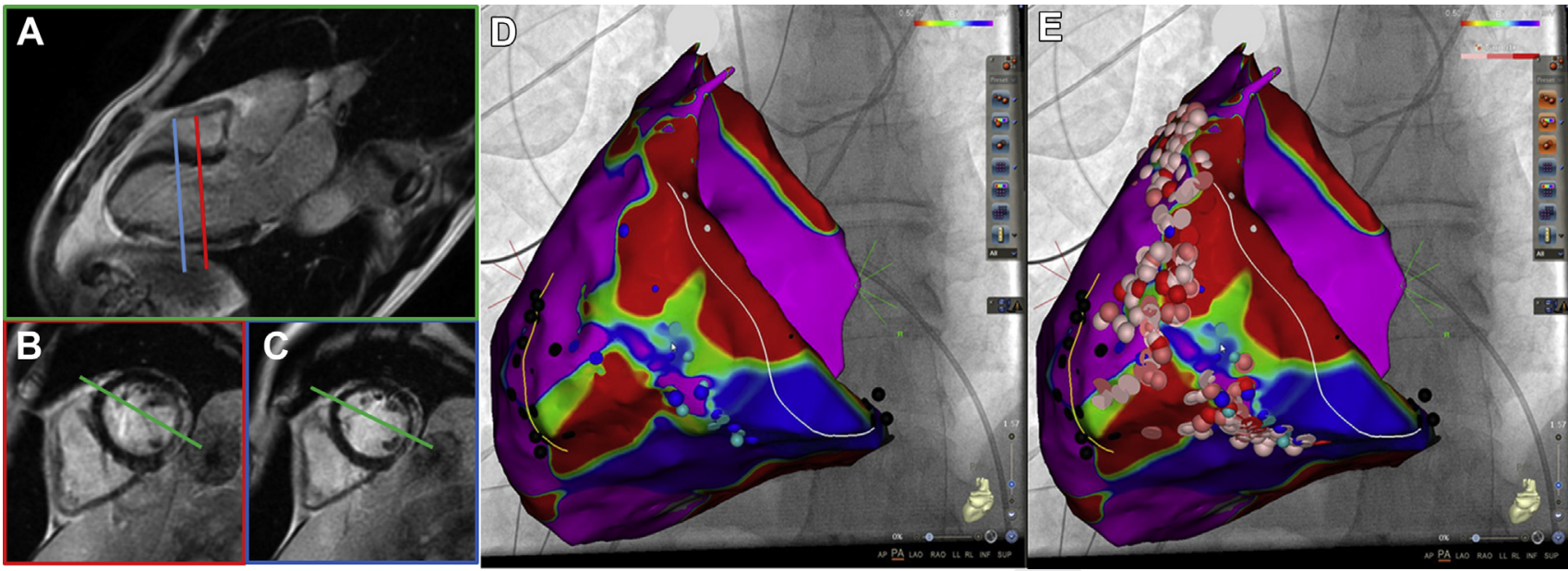

Figure 2 Inferolateral scar. A-C: Cardiac magnetic resonance long-axis $(\mathbf{A})$ and short-axis $(\mathbf{B}, \mathbf{C})$ views show hyperintensity signal on left ventricular posterolateral wall, suggestive of previous myocarditis. D, E: Epicardial bipolar voltage mapping (posteroanterior views) merged with fluoroscopy before (D) and after (E) catheter ablation. Black dots represent phrenic nerve. Blue and light-blue dots represent late and fragmented potentials. White to red dots represent ablation sites on the scar border.

\section{Procedural findings}

After clinical and invasive assessment, 61 patients (42.4\%) underwent CA: $43(70.5 \%)$ by an endocardial-only approach and 18 by an endocardial and adjunctive epicardial approach. The epicardial approach was used only in cases of sustained
VT and NSVT ablation (42 procedures), corresponding to $42.8 \%$ of all VT ablation procedures. Procedural data are given in Supplemental Table S3. Following CA, the number of AADs (particularly amiodarone) was reduced significantly and was lower in the CA than the AAD group ( 0.93 vs $1.70 ; P$

Table 1 Baseline characteristics of the general population according to management strategy

\begin{tabular}{|c|c|c|c|c|}
\hline & Total $(\mathrm{N}=144)$ & $\operatorname{AAD}(n=83[57.6 \%])$ & $C A(n=61[42.4 \%])$ & $P$ value \\
\hline Male sex & $83(57.6)$ & $48(57.8)$ & $37(60.7)$ & .73 \\
\hline Previous ICD implant & $48 / 134(35.8)$ & $25 / 73(34.2)$ & $23(37.7)$ & .68 \\
\hline PVCs & $48(33.3)$ & $33(39.8)$ & $15(24.6)$ & .07 \\
\hline NSVT & $28(19.4)$ & $18(21.7)$ & $10(16.4)$ & .52 \\
\hline LV EF (echocardiography) & $52.9 \pm 13$ & $52.6 \pm 13.8$ & $53.4 \pm 12.0$ & .74 \\
\hline LV EF $<50 \%$ & $39(27.1)$ & $22(26.5)$ & $17(40.1)$ & .10 \\
\hline LV EF $<35 \%$ & $11(7.6)$ & $7(8.4)$ & $4(6.6)$ & .68 \\
\hline CMR & $104(72.2)$ & $67(80.7)$ & $37(60.7)$ & .008 \\
\hline RV EDVi $\left(\mathrm{mL} / \mathrm{m}^{2}\right)$ & $92.3 \pm 30.1$ & $94.8 \pm 20.9$ & $102.3 \pm 22.8$ & .33 \\
\hline LV EDVi $\left(\mathrm{mL} / \mathrm{m}^{2}\right)$ & $113.9 \pm 40.3$ & $115.9 \pm 46.4$ & $110.7 \pm 28.5$ & .68 \\
\hline Anteroseptal scar (CMR) & $38(36.5)$ & $22(32.8)$ & $16(43.2)$ & .88 \\
\hline Anteroseptal scar (EVM) & $50(39.1)$ & $27(35.5)$ & $23(43.4)$ & .25 \\
\hline Anteroseptal scar (EVM and CMR) & $64(44.4)$ & $37(44.6)$ & $27(44.3)$ & .97 \\
\hline EVM & $129(89.6)$ & $76(91.6)$ & $53(86.9)$ & .36 \\
\hline Unipolar scar $\left(\mathrm{cm}^{2}\right)$ & $28(10.0-67.7)$ & $27.0(10.0-75.9)$ & $41(12.0-61.9)$ & .42 \\
\hline Unipolar scar (\%) & $12.5(4.7-34.9)$ & $11.6(4.4-35.9)$ & $17.3(4.8-26)$ & .31 \\
\hline Bipolar scar $\left(\mathrm{cm}^{2}\right)$ & $12.9(4.7-25.1)$ & $14.5(4.2-22.7)$ & $10.3(4.9-28.9)$ & .75 \\
\hline Bipolar scar (\%) & $6.4(2.4-13.4)$ & $6.6(2.3-15.4)$ & $6.2(2.3-11.9)$ & .396 \\
\hline
\end{tabular}

Values are given as mean $\pm S D, n(\%)$, or median (interquartile range) unless otherwise indicated. Cutoff values for dilation were referred to international standards.

$A A D=$ antiarrhythmic drugs; $C A=$ catheter ablation; $C M R=$ cardiac magnetic resonance; $E D V i=$ end-diastolic volume index; $E F=$ ejection fraction; $E M B=$ endomyocardial biopsy; EVM = electroanatomic voltage mapping; ICD = implantable cardioverter-defibrillator; LGE = late gadolinium enhancement; $\mathrm{LV}=$ left ventricle; NSVT = nonsustained ventricular tachycardia; $\mathrm{PVC}=$ premature ventricular complex; $\mathrm{RV}=$ right ventricle; $\mathrm{VF}=$ ventricular fibrillation; $\mathrm{VT}=$ ventricular tachycardia. 
Table 2 Predictors of arrhythmic relapse in patients treated with CA

\begin{tabular}{llll}
\hline & \multicolumn{3}{l}{ Univariate analysis } \\
\cline { 2 - 5 } & HR & $95 \%$ CI & $P$ value \\
\hline Arrhythmia at presentation & & & \\
PVC & 0.96 & $0.27-3.4$ & .95 \\
NSVT & 0.71 & $0.16-3.15$ & .63 \\
$\quad$ Sustained VT & 0.92 & $0.33-2.53$ & .87 \\
$\quad$ VF or aborted sudden death & 2.44 & $0.53-11.2$ & .25 \\
Imaging data & & & \\
$\quad$ Reduced LV EF $(<50 \%)$ & 1.50 & $0.45-4.92$ & .508 \\
$\quad$ Reduced LV EF $(<35 \%)$ & $\mathbf{6 . 3 3}$ & $1.25-31.90$ & .025 \\
$\quad$ Anteroseptal scar & $\mathbf{3 . 6 0}$ & $1.14-11.39$ & $\mathbf{. 0 2 9}$ \\
$\quad$ LGE+ & 2.04 & $0.71-5.85$ & .19 \\
Procedural data & & & \\
$\quad$ Endocardial-epicardial procedure & 1.16 & $0.36-3.71$ & .80 \\
$\quad$ VT inducibility & $\mathbf{0 . 1 0}$ & $0.02-0.55$ & $\mathbf{. 0 0 7}$ \\
VT noninducibility after CA & 0.51 & $0.09-2.68$ & .43 \\
\hline
\end{tabular}

Cox proportion univariate and multivariate hazard analysis for predictors of VA-free survival in patients who underwent CA.

$\mathrm{CI}=$ confidence interval; $\mathrm{HR}=$ hazard ratio; other abbreviation as in Table 1.

$=.003)($ Supplemental Table S1). During the index hospitalization, 10 more patients underwent ICD insertion.

\section{Anteroseptal vs nonanteroseptal scar}

Baseline characteristics of patients with and those without anteroseptal involvement referred for CA were not significantly different with regard to clinical characteristics, arrhythmic presentation, imaging, and EVM data (Supplemental Table S4). Patients without anteroseptal involvement more frequently underwent epicardial mapping/ablation $(P=$ .025 ) (Table 2). Of note, CA acute procedural success (noninducibility) was similar for $\mathrm{S}+$ and $\mathrm{S}-$ patients $(81.8 \%$ vs $75.0 \%$, respectively; $P=.66$ ).

\section{Long-term follow-up}

The percentage of patients who successfully underwent adequate follow-up at 3, 6, 9, and 12 months was $95.1 \%$, $90.3 \%, 84.0 \%$, and $77.8 \%$, respectively. During median follow-up of 24.5 months (11.1-68.5), 51 patients (39.8\%) experienced VA recurrence. In the CA group, VA-free survival at 1,2, and 3 years was $87 \%, 77 \%$, and $70 \%$, respectively. In patients treated medically, VA free-survival was $68 \%, 58 \%$, and 53\%, respectively (Figure 2). During median follow-up of 26.9 months (15.9-91.6), 6 patients died, with mortality of $2 \%$ at 1 year and $4 \%$ at 2 years. There were no significant differences between the 2 groups $(\log$-rank $P=$ $.56)$.

Analyzing predictors of VA recurrence in patients undergoing CA, reduced LV function (LVEF $<35 \%$ ) (hazard ratio [HR] 6.33) and anteroseptal scar location (HR 3.60) was shown to be an independent predictor of arrhythmic relapse on univariate analysis (Table 2 and Figure $3 \mathrm{~A}$ ). With regard to predictors of VA recurrence in the overall population, reduced EF and anteroseptal scar were associated with arrhythmic relapse (HR 7.24 and 2.02, respectively), whereas CA conferred lower risk (HR 0.5) (Figure 3B and Table 3). Sudden cardiac death or ICD shock at presentation were also associated with higher risk of recurrence (HR 2.0) (Supplemental Figure S3A). Considering only patients referred for VT ablation, the presence of anteroseptal scar was associated with a trend toward higher recurrences, although this did not reach statistical significance (Supplemental Figure S3E).

\section{Procedural complications}

A total of 6 procedural complications (9.8\%) occurred. Three patients developed vascular access-related complications. Specifically, 2 patients developed arteriovenous fistula, without hemodynamic impact, and were managed conservatively. One patient developed femoral pseudoaneurysm requiring surgical repair. Two patients developed pericardial effusion without signs of tamponade and were managed with anti-inflammatory drugs. One patient developed liver hematoma and retroperitoneal hemorrhage secondary to pericardial puncture. He underwent abdominal surgery and recovered well. Full data are given in Supplemental Table S5.

\section{Discussion \\ Main findings}

This study describes the largest cohort of patients with prior myocarditis and VA reported to date. One of the major strengths of our study is the high number of EMBs performed. Prior myocarditis was EMB-proven in nearly $80 \%$ of cases, which give us high confidence when ruling out phenocopies such as idiopathic dilated cardiomyopathy and arrhythmogenic cardiomyopathy. The main findings of the study are as follows. (1) Irrespective of the treatment strategy (AAD or CA), we report a worse outcome in patients with anteroseptal scar, consistent with the results of previous studies analyzing patients with NICM and acute myocarditis. (2) In adequately selected patients, CA of VA in patients with prior myocarditis is a safe and effective approach to achieve longterm arrhythmia control. Our findings confirm and extend the results of previous studies showing how CA provides good long-term outcomes. ${ }^{8}$ (3) CA allowed achievement of good arrhythmic control with limited use of AADs, especially amiodarone, which was discontinued in $65.6 \%$ of patients. This is a particularly attractive goal considering the young age of our patients.

\section{Prior myocarditis: A specific subtype of NICM}

Data on VA ablation in patients with myocarditis are scarce. ${ }^{1,7,8,23-27}$ These patients often are reported under the broader category of NICM. Patients labeled as having NICM encompass a heterogeneous cohort. ${ }^{28,29}$ Different etiologies are associated with different arrhythmic substrates, markedly variable outcomes, and different long-term clinical implications. ${ }^{1,30}$ Patients with prior myocarditis represent a small $(6 \%)$ but significant percentage of such patients. ${ }^{30}$ 

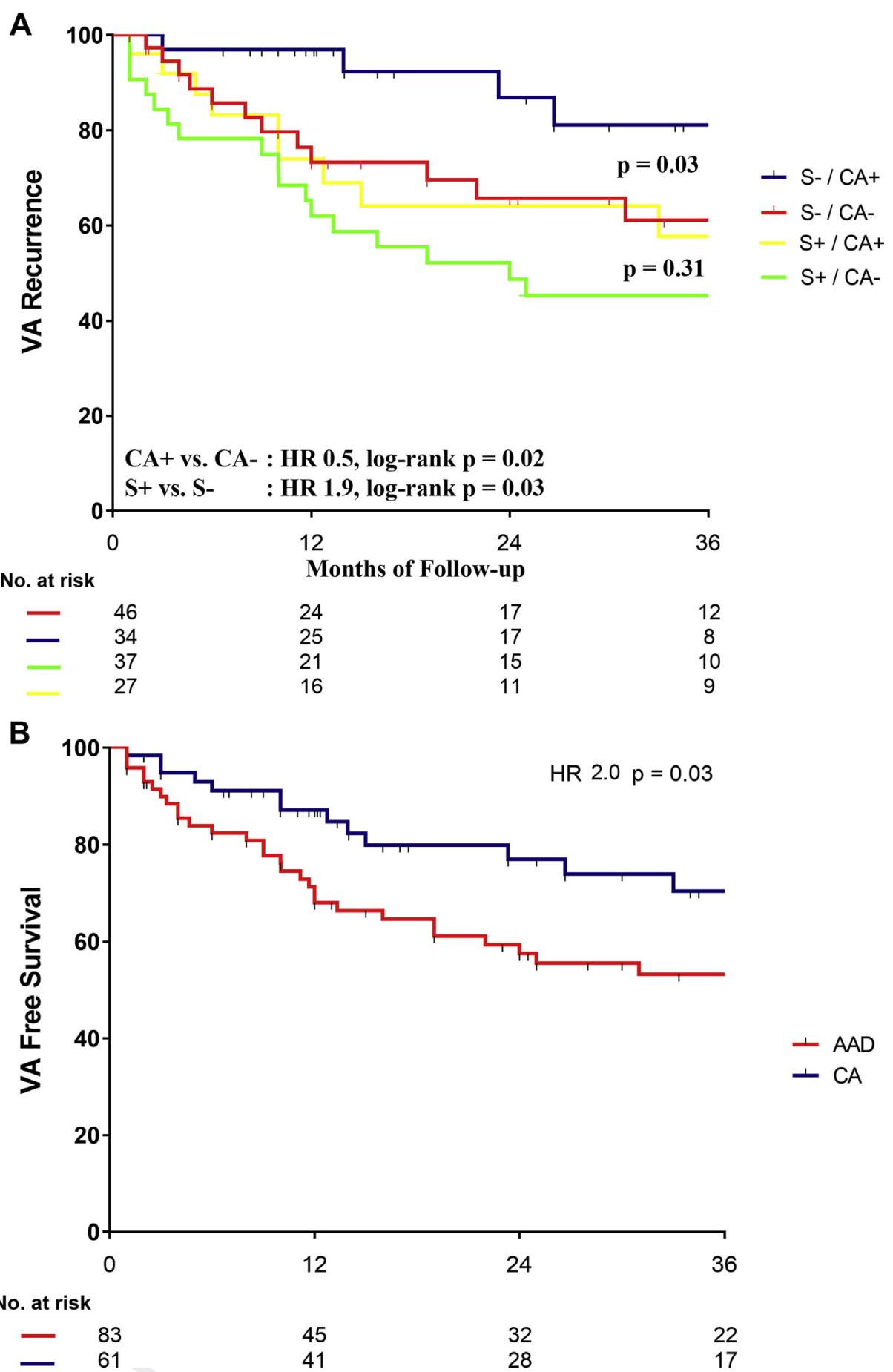

Figure 3 A: Kaplan-Meier curve shows how catheter ablation (CA) was superior to escalated antiarrhythmic drug therapy (AAD) in patients without septal scar (S-) (hazard ratio [HR] 0.3; $\log$-rank $P=.03$ ) but not in those with anteroseptal involvement $(\mathrm{S}+)(\mathrm{HR} 0.7$; $\log$-rank $P=.31)$. Overall, patients with $\mathrm{S}+$, irrespective of management strategy, had a higher recurrence rate on multivariate analysis (HR 1.9; log-rank $P=.03$ ). B: Kaplan-Meier survival curve shows ventricular arrhythmia (VA)-free survival in the overall population, stratified by treatment strategy: CA or medical therapy (ADD). CA seemed superior to AAD (HR $0.502[0.274-0.918] ; P=.025)$.

Compared to the majority of patients with NICM, those with myocarditis differ in 2 important aspects. First, they often have preserved EF, as confirmed in our cohort of patients. ${ }^{27}$ Second, the long-term outcome of postmyocarditis patients is better than in those wits other NICMs (eg, valvular cardiomyopathy, sarcoidosis, hypertrophic cardiomyopa- thy). ${ }^{30}$ Our results are in line with previous small cohort studies reporting $10 \%$ to $25 \%$ VA recurrence during median follow-up of 23-28 months for patients with myocarditis. $^{27-30}$

Of note, the 2019 HRS/EHRA/APHRS/LAHRS (Heart Rhythm Society/European Heart Rhythm Association/Asia 
Table 3 Predictors of arrhythmic relapse in the overall population

\begin{tabular}{llll}
\hline & \multicolumn{3}{l}{ Univariate analysis } \\
\cline { 2 - 4 } & HR & $95 \%$ CI & $P$ value \\
\hline Clinical presentation & & & \\
PVC & 0.90 & $0.50-1.63$ & .74 \\
NSVT & 1.04 & $0.53-2.04$ & .91 \\
Sustained VT & 0.95 & $0.24-1.07$ & .08 \\
$\quad$ Syncope or aborted & $\mathbf{2 . 0}$ & $1.11-3.64$ & .02 \\
$\quad$ sudden death & & & \\
Imaging data & & & \\
$\quad$ Reduced LV EF (<50\%) & 1.83 & $0.99-3.39$ & .053 \\
$\quad$ Reduced LV EF $(<35 \%)$ & $\mathbf{7 . 2 4}$ & $3.24-16.19$ & .001 \\
$\quad$ Anteroseptal scar & $\mathbf{2 . 0 2}$ & $1.15-3.54$ & .015 \\
$\quad$ LGE & 1.72 & $0.96-3.06$ & .068 \\
Catheter ablation & $\mathbf{0 . 5 0}$ & $\mathbf{0 . 2 7 - 0 . 9 2}$ & $\mathbf{. 0 2 5}$ \\
\hline
\end{tabular}

Cox proportion univariate and multivariate hazard analysis for predictors of VA-free survival in the overall population.

Abbreviations as in Tables 1 and 2.

Pacific Heart Rhythm Society/Latin American Heart Rhythm Society) Expert Consensus Statement on Catheter Ablation of Ventricular Arrhythmias and previous European and American guidelines did not specifically address the issue of VA ablation in patients with prior myocarditis. They simply recommend supportive therapy in the acute phase, and antiarrhythmic drugs and ICD insertion in cases of VA persistence. ${ }^{3,4,31}$ The absence of specific indications reflects the lack of high-quality evidence on this issue. ${ }^{8,24,25,27}$ The cumulative evidence arising from previous studies and from our own supports the concept that, in selected patients (ie, with refractory monomorphic VA at baseline and without anteroseptal scar), CA may be effective in achieving VA-free survival.

\section{Importance of scar pattern}

In our analysis, the location of the scar detected via LGE at magnetic resonance imaging and EVM was paramount in defining ablation success. Different scar locations and scar amounts carry different prognoses. ${ }^{12}$ In particular, in patients with NICM, 2 major categories are recognized: anteroseptal scar and nonseptal substrate with predominant basal inferolateral scar. ${ }^{9,32,33} \mathrm{~S}+$ is more often transmural than $\mathrm{S}_{-}$, which usually is subepicardial. ${ }^{33}$ However, the concept of scar location in patients with prior myocarditis undergoing CA of VA has never been specifically addressed. Interestingly, acute procedural success (noninducibility) was similar for $\mathrm{S}+$ and $\mathrm{S}-$ patients, whereas the rate or recurrence was $37 \%$ vs $13 \%$, respectively, at 2 years $(P=.03)$. These results are in line with a previous study from Oloriz et $\mathrm{al}^{9}$ of patients with NICM. ${ }^{33}$ For the first time, even in patients with prior myocarditis, our data support the notion that anteroseptal involvement of the arrhythmic substrate confers upon patients, irrespective of the treatment strategy, a high recurrence rate and poor procedural success. Specifically, in-depth localization of the critical isthmus is being sought as the main cause of the poor success of conventional unipolar CA. Alternative approaches (eg, alcohol ablation, bipolar ablation, radiotherapy) are under evaluation. ${ }^{34}$ Our findings suggest that optimal patient selection is a cornerstone for VT ablation success in patients with prior myocarditis.

With regard to clinical management of these patients, it is interesting to consider a recent paper by Simon et $\mathrm{al}^{12}$ analyzing the long term-follow-up (10 years) of 183 biopsy-proven cases of viral myocarditis. They confirmed that the presence of LGE in the anteroseptal segments was associated with higher mortality; however, they did not provide the mechanism behind the increased rate of death. The answer can instead be found in our study, as the increased rate of malignant VAs may explain, with longer-term follow-up, the higher mortality rate. Overall, although prospective data are lacking, the present body of evidence supports a more cautious management of patients with anteroseptal scar, with close follow-up. In addition, when in doubt about ICD insertion, this pattern might be taken into consideration.

Interestingly, in our population, anteroseptal scar was present in $44 \%$ of patients, which is slightly higher than in previously reported studies $(36 \%) .^{10-12}$ However, it has to be noted that anteroseptal involvement is associated with higher arrhythmic burden. If we consider that only patients referred for VA were enrolled, this explains the higher prevalence of anteroseptal involvement in our population.

\section{Study limitations}

Our study was retrospective, so proper comparison between the 2 management strategies (AAD and CA) is limited by selection bias. Indeed, our aim was to show the recurrence rate in each subgroup and not to compare 2 different strategies. We wanted to focus on real-world patients with a wide variety of VAs and to understand how, despite receiving best guideline-directed medical treatment, the scar pattern influenced outcome. Additionally, we report data from 2 specialized tertiary referral centers for VT ablation, so results may not be applicable to all institutions.

We expected use of the epicardial approach to improve VA-free survival. The fact that this finding did not emerge from our study may be secondary to the limited statistical power of the study.

With regard to follow-up, an ascertainment bias is present, as patients with an ICD were more subject to asymptomatic VA detection than patients who underwent Holter ECG monitoring. However, the number of patients with an ICD was not different between the 2 groups.

\section{Conclusion}

In a large cohort of patients with prior myocarditis and VA, anteroseptal scar was independently associated with VA recurrence, irrespective of the treatment strategy (medical therapy or CA). In adequately selected patients, CA conferred good VA-free survival at 2-year follow-up (77\%) with limited need for AADs. Our findings suggest that optimal patient selection is a cornerstone for VT ablation success in patients with prior myocarditis. 


\section{Acknowledgments}

We would like to thank Elena Sommariva and Selene Cellucci for their precious contribution to this paper.

\section{Appendix \\ Supplementary data}

Supplementary data associated with this article can be found in the online version at https://doi.org/10.1016/j.hrthm.202 0.12 .016 .

\section{References}

1. Peretto G, Sala S, Rizzo S, et al. Ventricular arrhythmias in myocarditis: characterization and relationships with myocardial inflammation. J Am Coll Cardiol 2020;75:1046-1057.

2. Maron BJ, Udelson JE, Bonow RO, et al. Eligibility and disqualification recommendations for competitive athletes with cardiovascular abnormalities: Task Force 3: hypertrophic cardiomyopathy, arrhythmogenic right ventricular cardiomyopathy and other cardiomyopathies, and myocarditis: a scientific statement from the American Heart Association and American College of Cardiology. J Am Coll Cardiol 2015;66:2362-2371.

3. Al-Khatib SM, Stevenson WG, Ackerman MJ, et al. 2017 AHA/ACC/HRS guideline for management of patients with ventricular arrhythmias and the prevention of sudden cardiac death: executive summary: a report of the American College of Cardiology/American Heart Association Task Force on Clinical Practice Guidelines and the Heart Rhythm Society. Heart Rhythm 2018;15:e190-e252.

4. Priori SG, Blomstrom-Lundqvist C, Mazzanti A, et al. 2015 ESC guidelines for the management of patients with ventricular arrhythmias and the prevention of sudden cardiac death: The Task Force for the Management of Patients with Ventricular Arrhythmias and the Prevention of Sudden Cardiac Death of the European Society of Cardiology (ESC). Endorsed by: Association for European Paediatric and Congenital Cardiology (AEPC). Eur Heart J 2015;36:2793-2867.

5. Caforio ALP, Pankuweit S, Arbustini E, et al. Current state of knowledge on aetiology, diagnosis, management, and therapy of myocarditis: a position statement of the European Society of Cardiology Working Group on Myocardial and Pericardial Diseases. Eur Heart J 2013;34:2636-2648. 2648a-2648d.

6. Kettering K, Kampmann C, Mollnau H, Kreitner K-F, Munzel T, Weiss C. Catheter ablation of an incessant ventricular tachycardia originating from the left aortic sinus cusp in an adolescent with subacute myocarditis. Clin Res Cardiol 2009;98:66-70.

7. Zeppenfeld K, Blom NA, Bootsma M, Schalij MJ. Incessant ventricular tachycardia in fulminant lymphocytic myocarditis: evidence for origin in the Purkinje system and successful treatment with ablation. Heart Rhythm 2007;4:88-91.

8. Dello Russo A, Casella M, Pieroni M, et al. Drug-refractory ventricular tachycardias after myocarditis: endocardial and epicardial radiofrequency catheter ablation. Circ Arrhythm Electrophysiol 2012;5:492-498.

9. Oloriz T, Silberbauer J, MacCabelli G, et al. Catheter ablation of ventricular arrhythmia in nonischemic cardiomyopathy: anteroseptal versus inferolateral scar sub-types. Circ Arrhythm Electrophysiol 2014;7:414-423.

10. Aquaro GD, Ghebru Habtemicael Y, Camastra G, et al. Prognostic value of repeating cardiac magnetic resonance in patients with acute myocarditis. J Am Coll Cardiol 2019;74:2439-2448.

11. Aquaro GD, Perfetti M, Camastra G, et al. Cardiac MR with late gadolinium enhancement in acute myocarditis with preserved systolic function: ITAMY Study. J Am Coll Cardiol 2017;70:1977-1987.

12. Simon G, Andreas S, MKA L, et al. Predictors of mortality in patients with biopsy-proven viral myocarditis: 10-year outcome data. J Am Heart Assoc 2020; 9:e015351.

13. Friedrich MG, Marcotte F. Cardiac magnetic resonance assessment of myocarditis. Circ Cardiovasc Imaging 2013;6:833-839.
14. Friedrich MG, Sechtem U, Schulz-Menger J, et al. Cardiovascular magnetic resonance in myocarditis: a JACC White Paper. J Am Coll Cardiol 2009; 53:1475-1487.

15. Schumm J, Greulich S, Wagner A, et al. Cardiovascular magnetic resonance risk stratification in patients with clinically suspected myocarditis. J Cardiovasc Magn Reson 2014;16:14

16. Corrado D, van Tintelen PJ, McKenna WJ, et al. Arrhythmogenic right ventricular cardiomyopathy: evaluation of the current diagnostic criteria and differential diagnosis. Eur Heart J 2019:41:1414-1429.

17. Towbin JA, McKenna WJ, Abrams DJ, et al. 2019 HRS expert consensus statement on evaluation, risk stratification, and management of arrhythmogenic cardiomyopathy. Heart Rhythm 2019;16:e301-e372.

18. Towbin JA, Lorts A, Jefferies JL. Left ventricular non-compaction cardiomyopathy. Lancet 2015;386:813-825.

19. Satoh H, Sano M, Suwa K, et al. Distribution of late gadolinium enhancement in various types of cardiomyopathies: significance in differential diagnosis, clinical features and prognosis. World J Cardiol 2014;6:585-601.

20. Sechtem U, Mahrholdt H, Vogelsberg H. Cardiac magnetic resonance in myocardial disease. Heart 2007;93:1520-1527.

21. Vaughan Williams EM. Classification of antidysrhythmic drugs. Pharmacol Ther B 1975;1:115-138.

22. Casella M, Dello Russo A, Bergonti M, et al. Diagnostic yield of electroanatomic voltage mapping in guiding endomyocardial biopsies. Circulation 2020; 142:1249-1260.

23. Zeppenfeld K. Ventricular tachycardia ablation in nonischemic cardiomyopathy. JACC Clin Electrophysiol 2018;4:1123-1140.

24. Mazzone P, Tsiachris D, Della Bella P. Epicardial management of myocarditisrelated ventricular tachycardia. Eur Heart J 2013;34:244.

25. Peretto G, Sala S, Rizzo S, et al. Arrhythmias in myocarditis: state of the art. Heart Rhythm 2019;16:793-801.

26. Berte B, Sacher F, Cochet $\mathrm{H}$, et al. Postmyocarditis ventricular tachycardia in patients with epicardial-only scar: a specific entity requiring a specific approach. J Cardiovasc Electrophysiol 2015;26:42-50.

27. Maccabelli G, Tsiachris D, Silberbauer J, et al. Imaging and epicardial substrate ablation of ventricular tachycardia in patients late after myocarditis. Europace 2014;16:1363-1372.

28. Dinov B, Fiedler L, Schönbauer R, et al. Outcomes in catheter ablation of ventricular tachycardia in dilated nonischemic cardiomyopathy compared with ischemic cardiomyopathy: results from the prospective Heart Centre of Leipzig VT Study. Circulation 2014:129:728-736.

29. Muser D, Santangeli P, Castro SA, et al. Long-term outcome after catheter ablation of ventricular tachycardia in patients with nonischemic dilated cardiomyopathy. Circ Arrhythm Electrophysiol 2016;9:1-11.

30. Vaseghi M, Hu TY, Tung R, et al. Outcomes of catheter ablation of ventricula tachycardia based on etiology in nonischemic heart disease: an international ventricular tachycardia ablation center collaborative study. JACC Clin Electrophysiol 2018;4:1141-1150

31. Cronin EM, Bogun FM, Maury P, et al. 2019 HRS/EHRA/APHRS/LAHRS expert consensus statement on catheter ablation of ventricular arrhythmias. J Arrhythm 2019:35:323-484

32. Haqqani HM, Tschabrunn CM, Tzou WS, et al. Isolated septal substrate for ventricular tachycardia in nonischemic dilated cardiomyopathy: incidence, characterization, and implications. Heart Rhythm 2011; 8:1169-1176.

33. Piers SRD, Tao Q, van Huls van Taxis CFB, Schalij MJ, van der Geest RJ, Zeppenfeld K. Contrast-enhanced MRI-derived scar patterns and associated ventricular tachycardias in nonischemic cardiomyopathy: implications for the ablation strategy. Circ Arrhythm Electrophysiol 2013;6:875-883.

34. Della Bella P, Peretto G, Paglino G, et al. Bipolar radiofrequency ablation for ventricular tachycardias originating from the interventricular septum: safety and efficacy in a pilot cohort study. Heart Rhythm 2020; $17: 2111-2118$ 